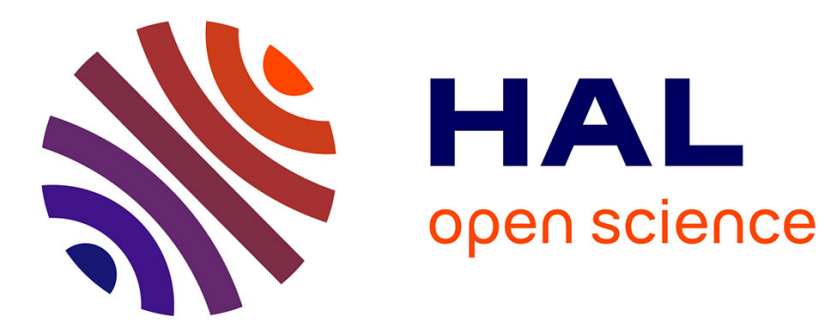

\title{
Heterochromatin establishment in the context of genome-wide epigenetic reprogramming.
}

\author{
Aline V Probst, Geneviève Almouzni
}

\section{To cite this version:}

Aline V Probst, Geneviève Almouzni. Heterochromatin establishment in the context of genome-wide epigenetic reprogramming.. Trends in Genetics, 2011, 27 (5), pp.177-85. 10.1016/j.tig.2011.02.002 . hal-00742753

\section{HAL Id: hal-00742753 \\ https://hal.science/hal-00742753}

Submitted on 17 Oct 2012

HAL is a multi-disciplinary open access archive for the deposit and dissemination of scientific research documents, whether they are published or not. The documents may come from teaching and research institutions in France or abroad, or from public or private research centers.
L'archive ouverte pluridisciplinaire HAL, est destinée au dépôt et à la diffusion de documents scientifiques de niveau recherche, publiés ou non, émanant des établissements d'enseignement et de recherche français ou étrangers, des laboratoires publics ou privés. 
Heterochromatin establishment in the context of genome-wide epigenetic reprogramming

Aline V. Probst ${ }^{1}$ and Geneviève Almouzni ${ }^{2, *}$

1 CNRS UMR 6247, INSERM U931, GReD, Clermont University, 24 Avenue des Landais, 63177 Aubière Cedex, France.

${ }^{2}$ Laboratory of Nuclear Dynamics and Genome Plasticity, Unité Mixte de Recherche 218 Centre National de la Recherche Scientifique/Institut Curie, 26, rue d'Ulm, 75248 Paris Cedex 05, France.

*Corresponding author: genevieve.almouzni@curie.fr 


\section{Abstract:}

Heterochromatin at pericentric satellites, characterized by a specific chromatin signature and chromocenter organization, is of paramount importance for genome function. Reestablishment of this organization after fertilization occurs in the context of genome-wide epigenetic reprogramming. Here, we summarize how the asymmetry in histone variants and post-translational modifications between paternal and maternal genomes and their respective pericentric heterochromatin domains evolves during early cleavage stages in mouse. We draw a parallel between these data and the burst of pericentric satellite transcription that occurs concomitantly with the dynamic reorganization of the pericentric domains into chromocenters in 2-cell embryos. Based on this new angle, we propose that a critical developmental transition at the 2-cell stage allows chromocenter formation by involving non-coding satellite transcripts to trigger specific chromatin changes.

Words: 123 


\section{The challenge of heterochromatin establishment}

The classical definition of heterochromatin states that, in opposition to euchromatin, heterochromatin corresponds to a chromosomal region that remains condensed through the cell cycle ${ }^{1}$. Within heterochromatin, transcription is generally repressed and heterochromatinization is critical for the transcriptional silencing of repetitive and transposable elements. In mouse, the predominant heterochromatin domain that is discernable by cytological techniques is pericentric heterochromatin ${ }^{2}$. Essentially composed of AT-rich (major) satellites (Figure 1a), which are arranged in tandem arrays of up to 2 megabases in length ${ }^{3,4}$, pericentric heterochromatin domains from different chromosomes cluster to form chromocenters in interphase (Figure 1b). This particular architecture is found in most somatic cells, but the degree of clustering and the positioning of the chromocenters in the nucleus can vary between cell types ${ }^{5,6}$. Pericentric heterochromatin flanks the centric domain where kinetochores form and has been proposed to play a role in chromatid cohesion and proper chromosome segregation.

At the molecular level, pericentric heterochromatin is characterized by specific epigenetic marks (Figure 2a) including DNA methylation and repressive posttranslational modifications. H3K9 trimethylation, which depends on the histone methyltransferases Suv39h1 and $2^{7}$, is the classic example of such a repressive mark. This histone mark is bound by the three isoforms of Heterochromatin Protein 1 $(\mathrm{HP} 1 \alpha, \beta, \gamma)^{8,9}$, which in turn recruit the H4K20 histone methyltransferases ${ }^{10}$ and DNA methyltransferases ${ }^{3}$ to establish a transcriptionally repressed chromatin state. Retention of HP1 and spreading of heterochromatin is largely ensured by a self-sustaining loop 
that entails both the self-association of HP1 into a flexible homodimer and its ability to interact with Suv39h ${ }^{11,12}$. Maintenance of this particular chromatin signature is required for proper centromere function since mice deficient in Suv39h methyltransferases lose H3K9me3 and HP1 at pericentric domains and show chromosome missegregation ${ }^{7}$.

During the cell cycle, in particular following replication, epigenetic marks are diluted after the passage of the replication fork. Therefore, sophisticated mechanisms that exploit the mutual reinforcement of DNA and histone modifications are required to ensure inheritance of chromatin marks and maintenance of pericentric heterochromatin 12-14. Besides these maintenance mechanisms, one should also consider how heterochromatin is established de novo at pericentric satellites. Establishment takes place during the first cell cycles of development, which are characterized by genomewide epigenetic reprogramming. The most extreme situation is seen for paternal pericentric domains, since the highly specialized male gametes lose somatic heterochromatin marks, which thus have to be regained after fertilization to ensure proper cellular divisions.

In this review, we concentrate on recent work regarding the establishment of heterochromatin during pre-implantation development in mouse. We will discuss how pericentric domains are reorganized from their gamete-specific structures and the important changes in chromatin organization undergone in particular by the paternal pericentric domains in the context of genome wide epigenetic reprogramming. We will then review recent evidence for an implication of a specific post-translational modification of $\mathrm{H} 3.3$ and non-coding transcripts in the decisive developmental transition during the 2-cell stage of embryonic development, during which chromocenters form for the first time. 


\section{Specific organization of pericentric domains acquired during gametogenesis}

Before two specialized gametes meet at fertilization, the chromatin of oocytes and spermatids has undergone important rearrangements during gametogenesis, which also extend to pericentric heterochromatin. During oogenesis, pericentric heterochromatin is remodeled, resulting in rings around the nucleolus in transcriptionally silent full-grown oocytes ${ }^{15}$. This particular organization is reiterated later in the zygote ${ }^{16}$ and correlates with the developmental competence of the embryo, as only oocytes with this heterochromatin arrangement develop beyond the 2-cell stage when fertilized in vitro ${ }^{17}$, 18. Despite the dissolution of chromocenters, pericentric domains in full-grown oocytes maintain, to some extent, a somatic heterochromatin signature: pericentric chromatin is enriched in $\mathrm{H} 3 \mathrm{~K} 9 \mathrm{me} 3$ and HP1 $\beta$ but lacks HP1 $\alpha^{19}$.

In contrast to the oocyte genome, the sperm genome contains small basic proteins, the protamines, which facilitate high-density DNA packaging, and replace histones on all but $1 \%$ of the mouse sperm DNA ${ }^{20}$. Recent evidence suggests that, at least in human, some limited paternal histone marks are transferred to the embryo. In both mouse and human, nucleosomes from regulatory regions of certain genes with important functions in embryonic development are retained in the oocyte ${ }^{21,22}$. At mouse pericentric heterochromatin domains, which cluster to a single chromocenter in the core of the sperm nucleus ${ }^{23}$, replacement of histones by protamines takes place progressively and with slower kinetics than in euchromatin ${ }^{24}$. Replacement is initiated by a wave of histone acetylation followed by the enrichment of specific histone $\mathrm{H} 2 \mathrm{~A}$ variants $\mathrm{H} 2 \mathrm{AL} 1 / 2$ at pericentric satellites ${ }^{25}$. These histone variants are retained in the 
pericentric domains of mature sperm heads, potentially forming a new DNA-packaging structure devoid of $\mathrm{H} 3 / \mathrm{H} 4$ dimers ${ }^{25}$. In addition, some nucleosomes containing histone $\mathrm{H} 4$ acetylated at position $\mathrm{K} 8$ and $\mathrm{K} 12$ co-localize in small discrete spots with the unique chromocenter in the sperm nucleus ${ }^{24}$. This suggests a role of acetylated $\mathrm{H} 4$ in packaging centric heterochromatin ${ }^{23,24}$, which also contains the centromere-specific $\mathrm{H} 3$ variant Cenp-A ${ }^{26}$. Thus, some chromatin-based structural information remains stable in centromere domains during spermatogenesis. One can hypothesize that the centromeric nucleosomal particle present at the centric domain can serve to promote the de novo establishment of correct heterochromatin features in the flanking pericentric domains, potentially through spreading of heterochromatin modifications. The immediate removal of the sperm-specific $\mathrm{H} 2 \mathrm{~A}$ variants that takes place following fertilization ${ }^{27}$ precludes their direct involvement in the re-establishment of somatic heterochromatin marks in the zygote. However, whether other architectural features or histone variants (i.e. Cenp-A) in the sperm impact chromatin organization in the embryo remains to be investigated.

In conclusion, important parental-specific reorganization affects pericentric domains during gametogenesis. While in the oocyte most of the somatic epigenetic heterochromatin marks are retained, male pericentric domains largely lose heterochromatin modifications. Therefore a de novo establishment of somatic heterochromatin features is required after fertilization in order to ultimately equalize the pericentric regions of distinct parental origins.

\section{Dynamics of histone variants and epigenetic marks during early cleavage stages}

Following fertilization, the oocyte completes meiosis II and extrudes one haploid genome in the form of the polar body, while the sperm genome decondenses. Two 
pronuclei form in the same cytoplasm, and the zygote resumes the first mitotic cell cycle. During the entire zygote stage, the two parental genomes remain as separate entities characterized by distinct chromatin signatures and transcriptional competence ${ }^{28-32}$. The two genomes finally align at the same metaphase plate during the first mitosis, but can be found spatially separated within the nucleus at least up to the 4-cell stage ${ }^{33-35}$. The reason for this spatial separation has remained largely unsolved; however, one plausible explanation could be that the separated genomes sustain differential reprogramming which therefore allows the extensive chromatin remodeling of the paternal genome following fertilization.

Once the sperm has entered the egg, protamines are removed immediately and are replaced by histones to form nucleosomal structures. Since histone incorporation takes place outside the S-phase, the replacement $\mathrm{H} 3$ variant $\mathrm{H} 3.3$, which can be incorporated in a DNA synthesis-independent manner, is used to package paternal DNA into nucleosomes ${ }^{35-38}$. Accordingly, the $\mathrm{H} 3.3$ histone chaperone histone regulator $\mathrm{A}$ (HIRA) is observed in the paternal pronucleus at the earliest stages ${ }^{36}$. However, in light of the identification of H3.3 deposition complexes including death domain associated protein DAXX and DEK ${ }^{39-41}$, the implication of other chaperones in $\mathrm{H} 3.3$ deposition at this moment in development should also be considered. The selective incorporation of $\mathrm{H} 3.3$ in the paternal genome results in asymmetry in histone $\mathrm{H} 3$ variants between the two parental genomes. Only a few hours later, during DNA replication, the canonical $\mathrm{H} 3$ variants are incorporated for the first time into the paternal genome.

In somatic cells, $\mathrm{H} 3.3$ is enriched at transcriptionally active regions ${ }^{42}$ and associates preferentially with histone post-translational modifications (PTMs) that mark "active" chromatin ${ }^{14}$. Accordingly, the paternal genome is initially hyperacetylated at the 
time of $\mathrm{H} 3.3$ incorporation ${ }^{28}$ and shows elevated transcriptional activity during the minor zygotic gene activation ${ }^{29-31}$. However $\mathrm{K} 4 \mathrm{me} 3$ on $\mathrm{H} 3.3$, another modification associated with gene activation, is lacking; this hints at embryo-specific functions of H3.3 independent of transcriptional activation ${ }^{43}$. The paternal pronucleus is further devoid of repressive histone marks such as $\mathrm{H} 3 \mathrm{~K} 9 \mathrm{me} 2$ and $\mathrm{me} 3^{32,44,45}$.

$\mathrm{H} 2 \mathrm{~A}$ variants are also uniquely distributed during pre-implantation development. In contrast to somatic cells, $\mathrm{H} 2 \mathrm{AZ}$, macroH2A and the canonical $\mathrm{H} 2 \mathrm{~A}$ are not or rather poorly incorporated into chromatin during the early cleavage stages and possibly even actively removed after fertilization ${ }^{46,47}$. In contrast, $\mathrm{H} 2 \mathrm{AX}$ is particularly enriched in early embryos and its specific C-terminal domain is responsible for the observed selective incorporation ${ }^{47}$. Furthermore, forced incorporation of $\mathrm{H} 2 \mathrm{AZ}$ or macroH2A as fusion protein with the C-terminal domain of $\mathrm{H} 2 \mathrm{AX}$, results in developmental retardation ${ }^{47}$. Therefore, the absence of $\mathrm{H} 2 \mathrm{AZ}$ and macroH2A is correlated with potency and potentially required for the dynamic changes in chromatin organization during early development. This hypothesis is in agreement with the observation that $\mathrm{H} 2 \mathrm{AZ}$ accumulates in differentiating cells of the inner cell mass (ICM) ${ }^{48}$ and is required for EScell differentiation ${ }^{49}$.

Reprogramming DNA methylation marks also reinforces the epigenetic asymmetry between the two genomes. The paternal genome, which is highly methylated ${ }^{50}$, shows a significant loss of DNA methylation during the first hours after fertilization ${ }^{51,}$ 52. The reprogramming occurs genome-wide and includes repetitive sequences like LINE elements and early retrotransposons ${ }^{53}$. Given the rapidity of this loss, active demethylation processes have been suggested ${ }^{52,54}$. Recent studies support this scenario, but suggest rather complex dynamic and sequence-dependent methylation 
changes involving single-stranded DNA breaks and base excision repair (BER) processes ${ }^{53,55}$ or the elongator complex to promote paternal demethylation ${ }^{56}$. The implication of DNA repair is supported by the presence of foci of the DNA damage marker $\gamma \mathrm{H} 2 \mathrm{~A} . \mathrm{X}$ in pre-replicative stages that are enhanced upon blockage of DNA polymerases ${ }^{53}$, and accumulation of BER pathway proteins in the male pronucleus ${ }^{55}$. The exact trigger for BER, however, remains to be determined. Deamination of 5methylcytosine $(5 \mathrm{mC})$ has long been suggested to play a role in DNA demethylation and recent data have shown an effect on methylation levels during the DNA reprogramming process in primordial germ cells (PGCs) in the absence of the Activation-induced cytidine deaminase (AID) ${ }^{57,58}$. An alternative trigger for BER could be the modification of $5 \mathrm{mC}$ to the recently identified intermediate 5 -hydroxymethylcytosine $(5 \mathrm{hmC})^{53,55,59-}$ ${ }^{61}$, which is then removed by a DNA glycosylase. Support for this possibility comes from (i) the difference in demethylation rates observed by $5 \mathrm{mC}$ antibodies and bisulfite sequencing, a technique that cannot distinguish between the two modifications, and (ii) the presence of Tet1, one of the enzymes catalyzing this reaction, in PGCs ${ }^{53,55,60}$. Interestingly, the maternal genome is protected from this initial active DNA demethylation by a process that involves the DNA-binding protein Stella (also known as PGC7) ${ }^{62}$. How this protein, which is present in both parental pronuclei, shields maternal DNA methylation globally and how it ensures the selective protection of DNA methylation at imprinted genes in the paternal pronucleus needs to be resolved. Likely candidates for selective recruitment of Stella to DNA are some maternal-specific histone modifications like $\mathrm{H} 3 \mathrm{~K} 9 \mathrm{me} 2$ and me ${ }^{32}$. Following the initial paternal-specific DNA methylation reprogramming, both parental genomes are further subjected to passive 
DNA demethylation during the subsequent cell cycles ${ }^{63}$ before the genome of ICM cells becomes specifically remethylated in blastocysts ${ }^{52}$.

Much has been speculated on the functional meaning of the epigenetic asymmetry between the two parental genomes. For example, it has been suggested that it could facilitate selective transcriptional activation of genes in the paternal or maternal genome and help avoid repeating reductional divisions in a cytoplasm programmed for meiosis ${ }^{32,64}$. The epigenetic asymmetry potentially contributes to keeping distinct chromatin states at loci subject to genomic imprinting and expressed in a parent-oforigin specific manner ${ }^{65}$. At other loci, however, like the pericentric satellite domains, the different chromatin features must be reconciled.

\section{Distinct chromatin signatures of the two parental pericentric domains}

The pericentric domains reorganize dynamically following fertilization to form rings around the nucleolar-like bodies (Figure $2 b$ and $3 a)^{16,66}$, similar to the organization in the oocyte nucleus. Given that this organization is achieved in normal and in parthenogenetic embryos, this supports a developmental stage-specific organization that is initially independent of the parental origin and the epigenetic marks of the parental genomes ${ }^{16,34}$. Even during reprogramming of embryonic stem (ES) or somatic cell nuclei in eggs, this arrangement of satellite repeats is repeated, suggesting that it is an essential hallmark of the genome-wide reprogramming process ${ }^{66,67}$. While in the zygote, few chromocenters are occasionally observed in the maternal pronucleus ${ }^{16}$, it is during the 2-cell stage that both parental pericentric domains progressively organize from ring structures into chromocenters (Figure 3a). The chromocenters are then retained during the remaining cleavage stage of pre-implantation development ${ }^{16,34}$. In 
addition to the maternal-paternal separation in 2-cell stage embryos, centromeres in most nuclei cluster to one side of the nucleus, adopting a configuration termed the Rabl configuration ${ }^{68}$. The significance of this spatial organization remains unclear but could reflect the chromosome arrangement in anaphase and exert certain constraints on nuclear organization at this developmental stage ${ }^{68,69}$.

While both parental pericentric satellites retain DNA methylation ${ }^{32,71}$, these domains reveal strikingly distinct properties at the level of their histone modifications in the zygote (Figure 2b). Maternal pericentric satellites are marked by H3K64me3, H3K9me3 and H4K2Ome3 and bind HP1 $\beta$ 16, 32, 70. HP1 $\alpha$, undetectable in the oocyte and the zygote ${ }^{19,31}$ only starts to be detected in 2-cell embryos ${ }^{34}$. This points to differential dynamics of these two HP1 isoforms. The paternal pericentric domains, which become enriched in the histone variant $\mathrm{H} 3.3^{35}$, lack the typical somatic marks including $\mathrm{H} 3 \mathrm{~K} 9 \mathrm{me} 3$. The low levels of HP1 $\beta$ observed at paternal pericentric satellites further underline an asymmetric distribution compared to the corresponding maternal regions ${ }^{16,32,35}$, and suggest the existence of an H3K9me3-independent localization. Thus, analyzing this time window should help to reveal interesting features of inheritance versus acquisition of the three HP1 isoforms during the earliest stages of development.

Instead of the typical pericentric heterochromatin modifications found in somatic cells, paternal pericentric domains are enriched in the alternative repressive chromatin factors, the Polycomb group binding proteins (Figure 2b). The Polycomb repressive complex 2 (PRC2) through its enzymatic activity responsible for trimethylation of H3K27me3 is required for PRC1 binding at certain genes ${ }^{71}$ and in general to 
euchromatin in embryos ${ }^{31}$. However the interdependency of the two complexes might be different at pericentric domains. While paternal pericentric domains in late zygotes acquire $\mathrm{H} 3 \mathrm{~K} 27 \mathrm{me} 3$ at the time of DNA replication ${ }^{35}, \mathrm{PRC} 1$ components are already found associated with paternal satellite repeats shortly after gamete fusion ${ }^{31}$. More importantly, embryos deficient for maternal and zygotic Enhancer of zeste homolog 2 (Ezh2), the methyltransferase responsible for $\mathrm{H} 3 \mathrm{~K} 27$ methylation, still specifically retain PRC1 components at paternal pericentric domains suggesting that mechanisms distinct from those in euchromatin operate at these domains to stabilize PRC1 proteins. In embryos deficient for the histone methyltransferase Suv39h2 both parental pericentric domains are devoid of H3K9me3. Interestingly, in this situation H3K27me3 and PRC1 are also found in maternal pericentric satellites. Therefore, Suv39h2-dependent somatic heterochromatin modifications block the accumulation of PRC1 complexes, which suggests that PRC1 might function as a back-up repressive system ${ }^{31}$. While methylation of $\mathrm{H} 3 \mathrm{~K} 27$ seems to be dispensable for PRC1 enrichment at paternal pericentric heterochromatin, the $\mathrm{H} 3$ position 27 lysine residue itself is critical for preimplantation development ${ }^{35}$. In agreement with the specific enrichment of $\mathrm{H} 3.3$ in pericentric heterochromatin ${ }^{35}$, the expression of a mutated form of $\mathrm{H} 3.3$, but not $\mathrm{H} 3.1$, carrying an arginine instead of a lysine at position 27 (K27R), induces developmental phenotypes. Expression of H3.3K27R leads to impaired development, relocalization of pericentric satellites away from the NLBs, loss of HP1 $\beta$ from pericentric domains and defective chromosome segregation. However, the way in which the expression of H3.3K27R, which accumulates specifically at paternal pericentric chromatin, also affects HP1 association to maternal domains remains to be elucidated. Nevertheless, paternal 
centromere function seems to be particularly compromised in embryos expressing H3.3K27R since the misaligned chromosomes are of paternal origin ${ }^{35}$.

Together, these observations imply that the histone H3.3 variant with particular modifications at its K27 residue is critical for proper pericentric heterochromatin organization. This is important during the early stages of mouse embryonic development, before the parental asymmetry is lost and pericentric domains become indistinguishable at the 8-cell stage ${ }^{31,69}$ (Figure 3a).

\section{Strand-specific expression of pericentric satellites and their role in chromocenter formation and embryonic development}

In recent years a role for non-coding RNA in heterochromatin establishment has emerged. In fission yeast, despite the transcriptional repressed state of heterochromatin, there is clear evidence that transcription of the pericentric outer repeats and their subsequent processing has a role in the establishment of heterochromatin ${ }^{72,} 73$. Specifically, mutant strains in components of the RNAinterference (RNAi) pathway, like the ribonuclease Dicer, show reduced levels of the critical heterochromatin marks, H3K9 methylation and the HP1 homologue Swi6, at the pericentric repeats ${ }^{74}$. The bidirectional transcription of the outer repeats is regulated during the cell cycle and occurs in a discrete time window in S-phase, most likely facilitated by dilution of heterochromatin marks as a consequence of replication of the domain ${ }^{75,76}$. These transcripts are processed to small RNAs, which then direct histone modifiers to the pericentric repeats to reestablish histone modifications, Swi6/HP1 binding and consequently transcriptional silencing. 
In mammals, non-coding RNA molecules have been implicated in establishment of different types of heterochromatin, for example, the Xist RNA on the inactive Xchromosome ${ }^{77}$ as well as Kcnq1ot1 ${ }^{78}$ and Air at imprinted loci. These long non-coding RNA molecules are thought to function either via formation of a transcriptionally silent compartment that excludes Polymerase II or through a direct recruitment of repressive chromatin modifiers to the chromatin locus ${ }^{79-82}$. At the pericentric domains, a yet unidentified RNA component is required for HP1 binding ${ }^{83,84}$, and non-coding major satellite transcripts of heterogeneous length transcribed by RNA Polymerase II have been found in ES and somatic cells ${ }^{3,85-89}$. While their transcription is cell cycle regulated, in contrast to fission yeast, no direct link could be established between the time of their expression and processing and maintenance of heterochromatin marks during the cell cycle ${ }^{13,89}$. However, the role of transcripts of pericentric major satellites in the initial establishment of heterochromatin organization had not been addressed. Recent studies have now shown that pericentric satellites are indeed transcribed during early cleavage stages ${ }^{31,34,35}$. Only limited amounts of transcripts were found in oocytes, suggesting that they are not stored like some other maternal transcripts, but are first transcribed during the minor zygotic gene activation in late zygotes. Major satellites then undergo a peak in expression at the 2-cell stage before being downregulated by the 4cell stage ${ }^{34}$ (Figure 3a). Even though the peak correlates with the major wave of gene activation, the observed burst followed by a rapid downregulation is particular to major satellites, as other non-coding RNAs such as TERRA behave in a distinct manner ${ }^{34}$. Remarkably, this peak in expression correlates with the dynamic rearrangement of pericentric satellites from ring-structures to chromocenters (Figure 3a), which implies a link between chromocenter formation and the observed expression dynamics ${ }^{16,34,66}$. 
In fission yeast, transcription of pericentric repeats occurs bidirectionally and in mammals both strands are transcribed ${ }^{88}$. Intriguingly, the analysis of transcripts with probes that distinguish between the Forward and Reverse strands revealed a strandspecific regulation of the two transcripts. A major peak in expression of the Forward strand during S-phase of the 2-cell stage is followed by expression of the Reverse strand in the G2 phase of the cell cycle. Expression of Forward transcripts takes place even in embryos arrested at the G1/S border. This expression pattern therefore underlies an intrinsic developmental clock rather than strictly depend on cell-cycle progression. The two transcripts differ in their localization, as the Forward transcripts coat parts of the pericentric domains, whereas the Reverse transcripts appear as distinct spots on the pericentric satellite rings (Figure 3b). Forward transcripts are expressed predominantly from the paternal genome and are accordingly reduced in parthenogenetic embryos in which both pronuclei are of female origin and therefore retain somatic heterochromatin modifications at pericentric satellites. Expression at paternal pericentric domains is potentially favored by the lack of somatic heterochromatin marks and the relative enrichment of $\mathrm{H} 3.3$ in paternal compared to maternal pericentric satellites ${ }^{35}$. Interestingly, one study found $\mathrm{H} 3.3$ at pericentric heterochromatin in mouse embryonic fibroblasts (MEFs), where it depends on DAXX ${ }^{39}$. Less H3.3 incorporation due to depletion of DAXX or H3.3 results in reduced pericentric satellite expression ${ }^{39}$. In contrast, absence of the $\mathrm{K} 27$ residue on $\mathrm{H} 3.3^{35}$ or lack of a functional PRC1 complex ${ }^{31}$ cause increased satellite expression in embryos. While the observed increase in expression in H3.3K27R expressing embryos is rather subtle, loss of PRC1 has a clear effect on satellite expression in agreement with the H3K27me3independent recruitment of the PRC1 complex ${ }^{31}$. 
Interestingly, depletion of major satellite transcripts by microinjection of Locked Nucleic Acid (LNA)-DNA gapmers during the first two cell cycles of embryonic development results in developmental arrest during the G2 phase at the 2-cell stage. The arrested embryos fail to complete the reorganization of their pericentric satellites into chromocenters, thus supporting the presence of active mechanisms contributing to chromocenter clustering and the functional relevance of pericentric transcripts in this process. However, the exact mechanism by which pericentric satellite transcripts operate remains to be dissected. Given that, during the 2-cell stage, both transcripts are found within the same nucleus, they could potentially form double stranded RNA molecules, which could be further processed to trigger heterochromatin formation. Indeed, microinjection of a double stranded major RNA molecule can rescue developmental arrest and the lagging chromosome phenotype observed at the 2-cell stage when a mutant form of $\mathrm{H} 3.3 \mathrm{~K} 27$ is expressed ${ }^{35}$. Alternatively, major RNA could recruit and stabilize heterochromatin components.

An intriguing candidate for a chromatin protein to be recruited by major transcripts is HP1, which can bind RNA via its hinge domain ${ }^{84}$ and specifically interacts in a posttranslationally modified form with major Forward transcripts (Maison et al, in press). Recruitment of HP1, together with a histone methyltransferase, could then lead to spreading of heterochromatin marks ${ }^{12,13}$ and to the progressive replacement of PRC1 components by somatic histone modifications. Indeed, embryos expressing HP1 $\beta$ proteins that lack the hinge domain showed HP1 $\beta$ loss from pericentric satellites and chromosome segregation defects similar to embryos expressing the H3.3 K27R mutant form. In contrast, the chromodomain responsible for binding to $\mathrm{H} 3 \mathrm{~K} 9 \mathrm{me} 3$ is dispensable 
for proper HP1 $\beta$ localization ${ }^{35}$. Together, these data point towards a decisive developmental transition during the 2-cell stage of embryonic development, during which chromocenters form for the first time in development. This requires both the crucial K27 residue of $\mathrm{H} 3.3$ and likely a functional PRC1 complex, as well as regulated expression of the pericentric satellites, in order to ensure proper centromere function on the paternal genome.

Presently, it is unclear how the expression of major satellites is controlled in time and space and which promoters or transcription factors are responsible for the strandspecific expression patterns observed in the 2-cell stage embryo. Furthermore, the way in which this expression is rapidly downregulated at the end of the 2-cell stage and whether a combination of transcriptional and post-transcriptional silencing pathways is at work remain to be determined. While the formation of double-stranded intermediates of the two transcripts and their further processing is an attractive hypothesis, strandspecific probes reveal both distinct expression timing and localization and rarely showed co-localization of the two transcripts in the nucleus. However the possibility that under these experimental conditions double stranded RNA is undetectable cannot be excluded. Small amounts of double stranded pericentric transcripts or their degradation products, as shown in Schizosaccharomyces pombe ${ }^{73}$, might be sufficient to trigger heterochromatin formation, which is then propagated and consolidated in the subsequent cell cycles by RNA-independent mechanisms. Further work is clearly required to explore the possible connection between RNAi and heterochromatin establishment during mouse development. Given the specific interaction of HP1 with Forward transcripts and the requirement of the hinge domain for proper localization of 
HP1 $\beta$ in 2-cell embryos, recruitment of HP1 by nascent Forward transcripts to paternal chromatin is another possibility; however both pathways could function in parallel.

\section{Concluding remarks and future perspectives}

Important new findings have enabled us to define a critical developmental transition during which the organization of pericentromeric repeats in chromocenters is established. The importance, respectively, of major satellite transcription and incorporation of specific histone variants for proper pericentric heterochromatin organization opens up new avenues to understand the possible interdependency of these events. Future work should help identify how these events relate to other major rearrangements, including the sophisticated DNA demethylation mechanisms that are emerging, and determine key elements involved in the parental asymmetry. The situation observed in the mouse embryo provides an example of a major visible rearrangement of pericentric heterochromatin and it will be important to investigate whether similar principles are exploited in other situations where major nuclear reorganization affects these regions, such as in Rod cell photoreceptors or during

primordial germ cell reprogramming ${ }^{6,90}$. The parameters evidenced herein may thus prove valuable markers for genome wide reprogramming.

Words: 4216 


\section{Acknowledgments}

We apologize for not having been able to quote all colleagues for their contribution and thank M. Casanova, J. Clark and D. Filipescu for critical reading of the manuscript and M. Casanova for help with Figure 3.

\section{Glossary}

Heterochromatin: A chromosomal region that remains condensed throughout the cell cycle and that is characterized by a specific chromatin signature.

Constitutive Heterochromatin: Defined in opposition to facultative heterochromatin (e.g. the inactive $\mathrm{X}$-chromosome) as a chromosomal region that on both homologous chromosomes responds to heterochromatinization in the same way during development 91.

Pericentric Heterochromatin: A heterochromatic region adjacent to chromatin containing the centromere-specific histone $\mathrm{H} 3$ variant $\mathrm{CenH} 3$ and which is considered to be typical constitutive heterochromatin. However, recent evidence suggests that mouse pericentric heterochromatin does not comply with this definition from the earliest cleavage stages onwards ${ }^{31,32,92}$.

Chromocenter: A cluster of constitutive heterochromatin from different chromosomes that is formed during interphase

Pre-implantation development: Development of a mammalian embryo from the zygote to the blastocyst stage.

Reprogramming: The reversal of an epigenetic state, resulting in an altered cellular identity. In mammals, genome-wide reprogramming takes place in primordial germ cells (PGCs) and in the early embryo. 
Histone H3 variants: In mouse, five major histone $\mathrm{H} 3$ variants can be distinguished. The replicative variants $\mathrm{H} 3.1$ and $\mathrm{H} 3.2$ are expressed and assembled into chromatin during S-phase, the replacement variant $\mathrm{H} 3.3$ is assembled throughout the cell cycle ${ }^{38}$, and the centromeric protein A (CENP-A) during early G1-phase ${ }^{93}$. The H3t variant is testis specific.

DNA methylation: Covalent attachment of a methyl group to a DNA base, in general cytosine.

Epigenetic information: The classical definition for epigenetic information includes heritable information leading to changes in gene function that take place without alteration to the DNA sequence ${ }^{94}$. To date candidates for carriers of epigenetic information are chromatin modifications including DNA methylation, histone posttranslational information, histone variants, non-histone chromatin binding proteins, higher order organization and nuclear RNA (reviewed in ${ }^{13}$ ).

Polycomb group binding proteins: Repressive chromatin binding proteins, responsible for maintenance of cell identity found in two complexes (polycomb repressive complex 1 and 2).

\section{Figure legends}

Figure 1. Pericentric heterochromatin organization in somatic cells

(a) In the acrocentric chromosomes of Mus musculus the centromere localizes adjacent to the proximal telomere. The centromere consists of the pericentric (major satellites, red) and the centric (minor satellites) domain. While the kinetochore forms on centric 
chromatin, the adjacent pericentric heterochromatin contributes to centromere function by mediating chromatid cohesion.

(b) The pericentric heterochromatin domains (red) of each individual chromosome as can be seen in mitotic cells (left) cluster together with the corresponding regions from other chromosomes to form chromocenters in interphase (right). Images below show DNA fluorescence in situ hybridization (FISH) for major satellite repeats (red) in anaphase and interphase nuclei of mouse $3 T 3$ cells. DNA is stained with 4',6-diamidino-2- phenylindole (DAPI, grey). nuc = nucleolus, Scale bar, $10 \mu \mathrm{M}$.

Figure 2. Asymmetry in epigenetic marks at pericentric domains in the mouse zygote

(a) In somatic cells, pericentric heterochromatin is organized into chromocenters and shows a characteristic chromatin signature. Major satellite DNA (red) is highly methylated, enriched in $\mathrm{H} 2 \mathrm{AZ}{ }^{48}$, specific histone modifications and $\mathrm{HP} 1 \alpha$ and $\mathrm{HP} 1 \beta$ proteins $2,3,10,12$. The binding of HP1 to pericentric domains requires a structural RNA component ${ }^{83}$.

(b) During the first cell cycle of embryonic development, maternal and paternal pericentric satellite domains reveal distinct epigenetic signatures. Pericentric domains (red) form rings around the nucleolar-like bodies (NLBs) in both parental pronuclei. Chromocenters are occasionally observed in maternal pronuclei only ${ }^{16}$ and are retained in the polar body (PB). The maternal pericentric domains show somatic heterochromatin marks ${ }^{16,32,44}$ and transient enrichment in $\mathrm{H} 3 \mathrm{~K} 64 \mathrm{me} 3^{70}$. They bind HP1 $\beta$ however lack HP1 $\alpha^{32}$. Despite the genome-wide DNA demethylation of the paternal genome, DNA 
methylation is retained in the paternal pericentric rings ${ }^{51,95}$. Paternal pericentric domains are specifically enriched in the histone replacement variant $\mathrm{H} 3.3$, show H3K9me1, H3K27me1 and H3K27me3 and are enriched in proteins of the Polycomb repressive complex 1 , a repressive back-up system ${ }^{31}$. HP1 $\beta$ has been observed in some studies at paternal pericentric domains ${ }^{16,32}$, suggesting that HP1 $\beta$ might initially be recruited to paternal satellite DNA in a mechanism independent of H3K9me3.

\section{Figure 3. Dynamic reorganization and expression of pericentric satellites during pre-implantation development}

(a) During the first cleavage stages, pericentric heterochromatin dynamically reorganizes and undergoes a burst in transcription during which expression is regulated in a strand-specific manner. Brightfield images of embryos in culture are superimposed with schematics illustrating the organization of major satellites (pink) at the respective stages. Following fertilization, pericentric satellites organize into ring-structures around the nucleolar-like bodies. Chromocenter formation occurs during the 2-cell stage ${ }^{16,32,34}$ and is then propagated during further cleavage stages. Major events concerning histone variant incorporation and transcriptional activation (minor and major zygotic gene activation (ZGA) ${ }^{36,37,96}$ are indicated. The two parental genomes retain asymmetry in epigenetic marks up to the 8-cell stage. The transcription dynamics during early preimplantation development of the Forward (green) and the Reverse (red) major satellite transcripts are schematized below. Transcription peaks during chromocenter formation in 2-cell embryos and is downregulated in 4-cell embryos ${ }^{34}$. PB, polar body. 
(b) Images show early and late 2-cell embryos in which major Forward (green) and Reverse (red) satellite transcripts have been revealed by RNA FISH using LNA oligonucleotide probes that distinguish between the two transcripts ${ }^{34}$. Upper panels show maximum projections of the complete embryo and lower panels a magnification of one representative blastomere nucleus. DNA is counterstained with DAPI (grey), Scale bar, $10 \mu \mathrm{M}$. Note that the Forward transcripts are also found in the cytoplasm (arrow) and are highly expressed in early 2-cell embryos. By the late 2-cells stage Forward transcripts are downregulated, while Reverse transcripts are highly expressed.

\section{References}

1. Heitz, E. (1928) Das Heterochromatin der Moose. Jahrbuch Wiss Botanik, 762818

2. Guenatri, M., et al. (2004) Mouse centric and pericentric satellite repeats form distinct functional heterochromatin. J Cell Biol 166, 493-505

3. Lehnertz, B., et al. (2003) Suv39h-mediated histone H3 lysine 9 methylation directs DNA methylation to major satellite repeats at pericentric heterochromatin. Curr Biol 13, 1192-1200

4. Vissel, B., and Choo, K.H. (1989) Mouse major (gamma) satellite DNA is highly conserved and organized into extremely long tandem arrays: implications for recombination between nonhomologous chromosomes. Genomics 5, 407-414

5. Terranova, R., et al. (2005) The reorganisation of constitutive heterochromatin in differentiating muscle requires HDAC activity. Exp Cell Res 310, 344-356 
6. Solovei, I., et al. (2009) Nuclear architecture of rod photoreceptor cells adapts to vision in mammalian evolution. Cell 137, 356-368

7. Peters, A.H., et al. (2001) Loss of the Suv39h histone methyltransferases impairs mammalian heterochromatin and genome stability. Cell 107, 323-337

8. Bannister, A.J., et al. (2001) Selective recognition of methylated lysine 9 on histone H3 by the HP1 chromo domain. Nature 410, 120-124

9. Lachner, M., et al. (2001) Methylation of histone H3 lysine 9 creates a binding site for HP1 proteins. Nature 410, 116-120

10. Schotta, G., et al. (2004) A silencing pathway to induce H3-K9 and H4-K20 trimethylation at constitutive heterochromatin. Genes Dev 18, 1251-1262

11. Aagaard, L., et al. (1999) Functional mammalian homologues of the Drosophila PEV-modifier Su(var)3-9 encode centromere-associated proteins which complex with the heterochromatin component M31. Embo J 18, 1923-1938

12. Maison, C., and Almouzni, G. (2004) HP1 and the dynamics of heterochromatin maintenance. Nat Rev Mol Cell Biol 5, 296-304

13. Probst, A.V., et al. (2009) Epigenetic inheritance during the cell cycle. Nat Rev Mol Cell Biol 10, 192-206

14. Loyola, A., et al. (2006) PTMs on H3 variants before chromatin assembly potentiate their final epigenetic state. Mol Cell 24, 309-316

15. Zuccotti, M., et al. (1995) Chromatin organization during mouse oocyte growth. Mol Reprod Dev 41, 479-485

16. Probst, A.V., et al. (2007) Structural differences in centromeric heterochromatin are spatially reconciled on fertilisation in the mouse zygote. Chromosoma 116, 403-415 
17. Zuccotti, M., et al. (2002) The analysis of chromatin organisation allows selection of mouse antral oocytes competent for development to blastocyst. Zygote 10, 73-78

18. De La Fuente, R., et al. (2004) Major chromatin remodeling in the germinal vesicle (GV) of mammalian oocytes is dispensable for global transcriptional silencing but required for centromeric heterochromatin function. Dev Biol 275, 447-458

19. Meglicki, M., et al. (2008) Constitutive heterochromatin during mouse oogenesis: the pattern of histone $\mathrm{H} 3$ modifications and localization of HP1alpha and HP1beta proteins. Mol Reprod Dev 75, 414-428

20. Bench, G.S., et al. (1996) DNA and total protamine masses in individual sperm from fertile mammalian subjects. Cytometry 23, 263-271

21. Hammoud, S.S., et al. (2009) Distinctive chromatin in human sperm packages genes for embryo development. Nature 460, 473-478

22. Brykczynska, U., et al. (2010) Repressive and active histone methylation mark distinct promoters in human and mouse spermatozoa. Nat Struct Mol Biol 17, 679-687

23. Haaf, T., and Ward, D.C. (1995) Higher order nuclear structure in mammalian sperm revealed by in situ hybridization and extended chromatin fibers. Exp Cell Res $219,604-611$

24. van der Heijden, G.W., et al. (2006) Transmission of modified nucleosomes from the mouse male germline to the zygote and subsequent remodeling of paternal chromatin. Dev Biol 298, 458-469

25. Govin, J., et al. (2007) Pericentric heterochromatin reprogramming by new histone variants during mouse spermiogenesis. J Cell Biol 176, 283-294

26. Palmer, D.K., et al. (1990) The centromere specific histone CENP-A is selectively retained in discrete foci in mammalian sperm nuclei. Chromosoma 100, 32-36 
27. Wu, F., et al. (2008) Testis-specific histone variants H2AL1/2 rapidly disappear from paternal heterochromatin after fertilization. J Reprod Dev 54, 413-417

28. Adenot, P.G., et al. (1997) Differential H4 acetylation of paternal and maternal chromatin precedes DNA replication and differential transcriptional activity in pronuclei of 1-cell mouse embryos. Development 124, 4615-4625

29. Aoki, F., et al. (1997) Regulation of transcriptional activity during the first and second cell cycles in the preimplantation mouse embryo. Dev Biol 181, 296-307

30. Bouniol, C., et al. (1995) Endogenous transcription occurs at the 1-cell stage in the mouse embryo. Exp Cell Res 218, 57-62

31. Puschendorf, M., et al. (2008) PRC1 and Suv39h specify parental asymmetry at constitutive heterochromatin in early mouse embryos. Nat Genet $40,411-420$

32. Santos, F., et al. (2005) Dynamic chromatin modifications characterise the first cell cycle in mouse embryos. Dev Biol 280, 225-236

33. Mayer, W., et al. (2000) Spatial separation of parental genomes in preimplantation mouse embryos. J Cell Biol 148, 629-634

34. Probst, A.V., et al. (2010) A strand-specific burst in transcription of pericentric satellites is required for chromocenter formation and early mouse development. Dev Cell $19,625-638$

35. Santenard, A., et al. (2010) Heterochromatin formation in the mouse embryo requires critical residues of the histone variant H3.3. Nat Cell Biol

36. van der Heijden, G.W., et al. (2005) Asymmetry in histone H3 variants and lysine methylation between paternal and maternal chromatin of the early mouse zygote. Mech Dev 122, 1008-1022 
37. Torres-Padilla, M.E., et al. (2006) Dynamic distribution of the replacement histone variant $\mathrm{H} 3.3$ in the mouse oocyte and preimplantation embryos. Int J Dev Biol 50, 455461

38. Tagami, H., et al. (2004) Histone H3.1 and H3.3 complexes mediate nucleosome assembly pathways dependent or independent of DNA synthesis. Cell 116, 51-61

39. Drane, P., et al. (2010) The death-associated protein DAXX is a novel histone chaperone involved in the replication-independent deposition of H3.3. Genes Dev 24, $1253-1265$

40. Goldberg, A.D., et al. (2010) Distinct factors control histone variant H3.3 localization at specific genomic regions. Cell 140, 678-691

41. Elsaesser, S.J., and Allis, C.D. (2010) HIRA and Daxx Constitute Two Independent Histone H3.3-Containing Predeposition Complexes. Cold Spring Harb Symp Quant Biol

42. Ahmad, K., and Henikoff, S. (2002) The histone variant H3.3 marks active chromatin by replication-independent nucleosome assembly. Mol Cell 9, 1191-1200

43. Lepikhov, K., and Walter, J. (2004) Differential dynamics of histone H3 methylation at positions K4 and K9 in the mouse zygote. BMC Dev Biol 4, 12

44. Arney, K.L., et al. (2002) Histone methylation defines epigenetic asymmetry in the mouse zygote. Int J Dev Biol 46, 317-320

45. Liu, H., et al. (2004) Regulation of histone H3 lysine 9 methylation in oocytes and early pre-implantation embryos. Development 131, 2269-2280

46. Chang, C.C., et al. (2005) A maternal store of macroH2A is removed from pronuclei prior to onset of somatic macroH2A expression in preimplantation embryos. Dev Biol 278, 367-380 
47. Nashun, B., et al. (2010) Changes in the nuclear deposition of histone H2A variants during pre-implantation development in mice. Development 137, 3785-3794

48. Rangasamy, D., et al. (2003) Pericentric heterochromatin becomes enriched with H2A.Z during early mammalian development. Embo J 22, 1599-1607

49. Creyghton, M.P., et al. (2008) H2AZ is enriched at polycomb complex target genes in ES cells and is necessary for lineage commitment. Cell 135, 649-661

50. Farthing, C.R., et al. (2008) Global mapping of DNA methylation in mouse promoters reveals epigenetic reprogramming of pluripotency genes. PLoS Genet 4, e1000116

51. Mayer, W., et al. (2000) Demethylation of the zygotic paternal genome. Nature $403,501-502$

52. Santos, F., et al. (2002) Dynamic reprogramming of DNA methylation in the early mouse embryo. Dev Biol 241, 172-182

53. Wossidlo, M., et al. (2010) Dynamic link of DNA demethylation, DNA strand breaks and repair in mouse zygotes. Embo $\mathrm{J} 29,1877-1888$

54. Reik, W. (2007) Stability and flexibility of epigenetic gene regulation in mammalian development. Nature 447, 425-432

55. Hajkova, P., et al. (2010) Genome-wide reprogramming in the mouse germ line entails the base excision repair pathway. Science $329,78-82$

56. Okada, Y., et al. (2010) A role for the elongator complex in zygotic paternal genome demethylation. Nature 463, 554-558

57. Rai, K., et al. (2008) DNA demethylation in zebrafish involves the coupling of a deaminase, a glycosylase, and gadd45. Cell 135, 1201-1212 
58. Popp, C., et al. (2010) Genome-wide erasure of DNA methylation in mouse primordial germ cells is affected by AID deficiency. Nature 463, 1101-1105

59. Kriaucionis, S., and Heintz, N. (2009) The nuclear DNA base 5hydroxymethylcytosine is present in Purkinje neurons and the brain. Science 324, 929930

60. Tahiliani, M., et al. (2009) Conversion of 5-methylcytosine to 5hydroxymethylcytosine in mammalian DNA by MLL partner TET1. Science 324, 930-935 61. Ito, S., et al. Role of Tet proteins in $5 \mathrm{mC}$ to $5 \mathrm{hmC}$ conversion, ES-cell selfrenewal and inner cell mass specification. Nature 466, 1129-1133

62. Nakamura, T., et al. (2007) PGC7/Stella protects against DNA demethylation in early embryogenesis. Nat Cell Biol 9, 64-71

63. Reik, W., et al. (2001) Epigenetic reprogramming in mammalian development. Science 293, 1089-1093

64. Wiekowski, M., et al. (1997) Changes in histone synthesis and modification at the beginning of mouse development correlate with the establishment of chromatin mediated repression of transcription. J Cell Sci 110 ( Pt 10), 1147-1158

65. Surani, M.A. (2001) Reprogramming of genome function through epigenetic inheritance. Nature 414, 122-128

66. Martin, C., et al. (2006) Genome restructuring in mouse embryos during reprogramming and early development. Dev Biol 292, 317-332

67. Maalouf, W.E., et al. (2009) Trichostatin A treatment of cloned mouse embryos improves constitutive heterochromatin remodeling as well as developmental potential to term. BMC Dev Biol 9, 11

68. Rabl, C. (1885) Über Zellteilung. Morph Jb 10, 214-311 
69. Merico, V., et al. (2007) Epigenomic differentiation in mouse preimplantation nuclei of biparental, parthenote and cloned embryos. Chromosome Res

70. Daujat, S., et al. (2009) H3K64 trimethylation marks heterochromatin and is dynamically remodeled during developmental reprogramming. Nat Struct Mol Biol 16, 777-781

71. Bernstein, E., et al. (2006) Mouse polycomb proteins bind differentially to methylated histone $\mathrm{H} 3$ and RNA and are enriched in facultative heterochromatin. $\mathrm{Mol}$ Cell Biol 26, 2560-2569

72. Grewal, S.I., and Jia, S. (2007) Heterochromatin revisited. Nat Rev Genet 8, 3546

73. Halic, M., and Moazed, D. (2010) Dicer-independent primal RNAs trigger RNAi and heterochromatin formation. Cell 140, 504-516

74. Volpe, T.A., et al. (2002) Regulation of heterochromatic silencing and histone H3 lysine-9 methylation by RNAi. Science 297, 1833-1837

75. Chen, E.S., et al. (2008) Cell cycle control of centromeric repeat transcription and heterochromatin assembly. Nature 451, 734-737

76. Kloc, A., et al. (2008) RNA interference guides histone modification during the $\mathrm{S}$ phase of chromosomal replication. Curr Biol 18, 490-495

77. Masui, O., and Heard, E. (2006) RNA and protein actors in X-chromosome inactivation. Cold Spring Harb Symp Quant Biol 71, 419-428

78. Terranova, R., et al. (2008) Polycomb group proteins Ezh2 and Rnf2 direct genomic contraction and imprinted repression in early mouse embryos. Dev Cell 15, 668-679 
79. Chaumeil, J., et al. (2006) A novel role for Xist RNA in the formation of a repressive nuclear compartment into which genes are recruited when silenced. Genes Dev 20, 2223-2237

80. Nagano, T., et al. (2008) The Air noncoding RNA epigenetically silences transcription by targeting G9a to chromatin. Science 322, 1717-1720

81. Pandey, R.R., et al. (2008) Kcnq1ot1 antisense noncoding RNA mediates lineage-specific transcriptional silencing through chromatin-level regulation. Mol Cell 32, 232-246

82. Ponting, C.P., et al. (2009) Evolution and functions of long noncoding RNAs. Cell $136,629-641$

83. Maison, C., et al. (2002) Higher-order structure in pericentric heterochromatin involves a distinct pattern of histone modification and an RNA component. Nat Genet $30,329-334$

84. Muchardt, C., et al. (2002) Coordinated methyl and RNA binding is required for heterochromatin localization of mammalian HP1alpha. EMBO Rep 3, 975-981

85. Cohen, A.K., et al. (1973) Transcription of satellite DNA in mouse L-cells. Can J Biochem 51, 529-532

86. Gaubatz, J.W., and Cutler, R.G. (1990) Mouse satellite DNA is transcribed in senescent cardiac muscle. J Biol Chem 265, 17753-17758

87. Schoeftner, S., and Blasco, M.A. (2008) Developmentally regulated transcription of mammalian telomeres by DNA-dependent RNA polymerase II. Nat Cell Biol 10, 228236

88. Rudert, F., et al. (1995) Transcripts from opposite strands of gamma satellite DNA are differentially expressed during mouse development. Mamm Genome 6, 76-83 
89. Lu, J., and Gilbert, D.M. (2007) Proliferation-dependent and cell cycle regulated transcription of mouse pericentric heterochromatin. J Cell Biol 179, 411-421

90. Hajkova, P., et al. (2008) Chromatin dynamics during epigenetic reprogramming in the mouse germ line. Nature 452, 877-881

91. Brown, S.W. (1966) Heterochromatin. Science 151, 417-425

92. Probst, A.V., and Almouzni, G. (2008) Pericentric heterochromatin: dynamic organization during early development in mammals. Differentiation $76,15-23$

93. Jansen, L.E., et al. (2007) Propagation of centromeric chromatin requires exit from mitosis. J Cell Biol 176, 795-805

94. Riggs, A.D., et al. (1996) Epigenetic Mechanisms of Gene Regulation. 1-4

95. Rougier, N., et al. (1998) Chromosome methylation patterns during mammalian preimplantation development. Genes Dev 12, 2108-2113

96. Schultz, R.M. (2002) The molecular foundations of the maternal to zygotic transition in the preimplantation embryo. Hum Reprod Update 8, 323-331 


\section{(a) typical mouse acrocentric chromosome}

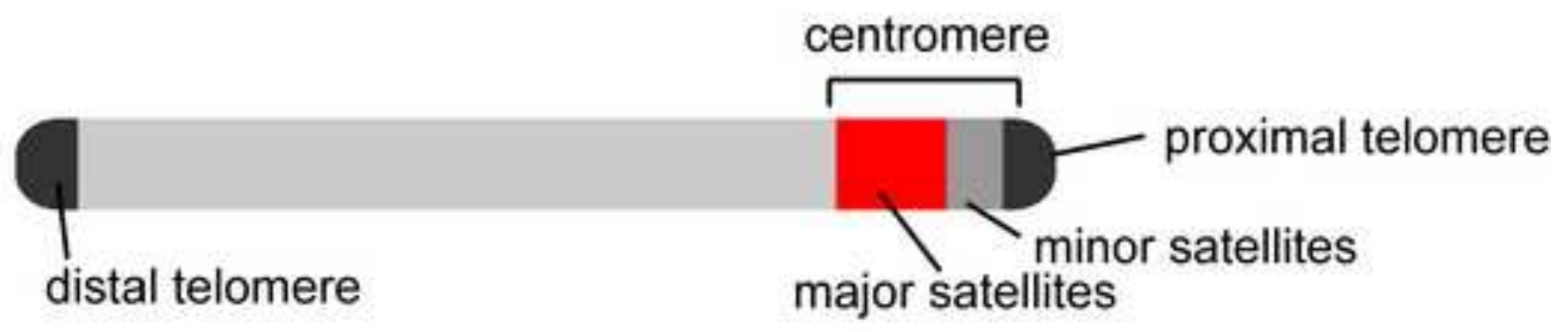

(b) chromocenter organization

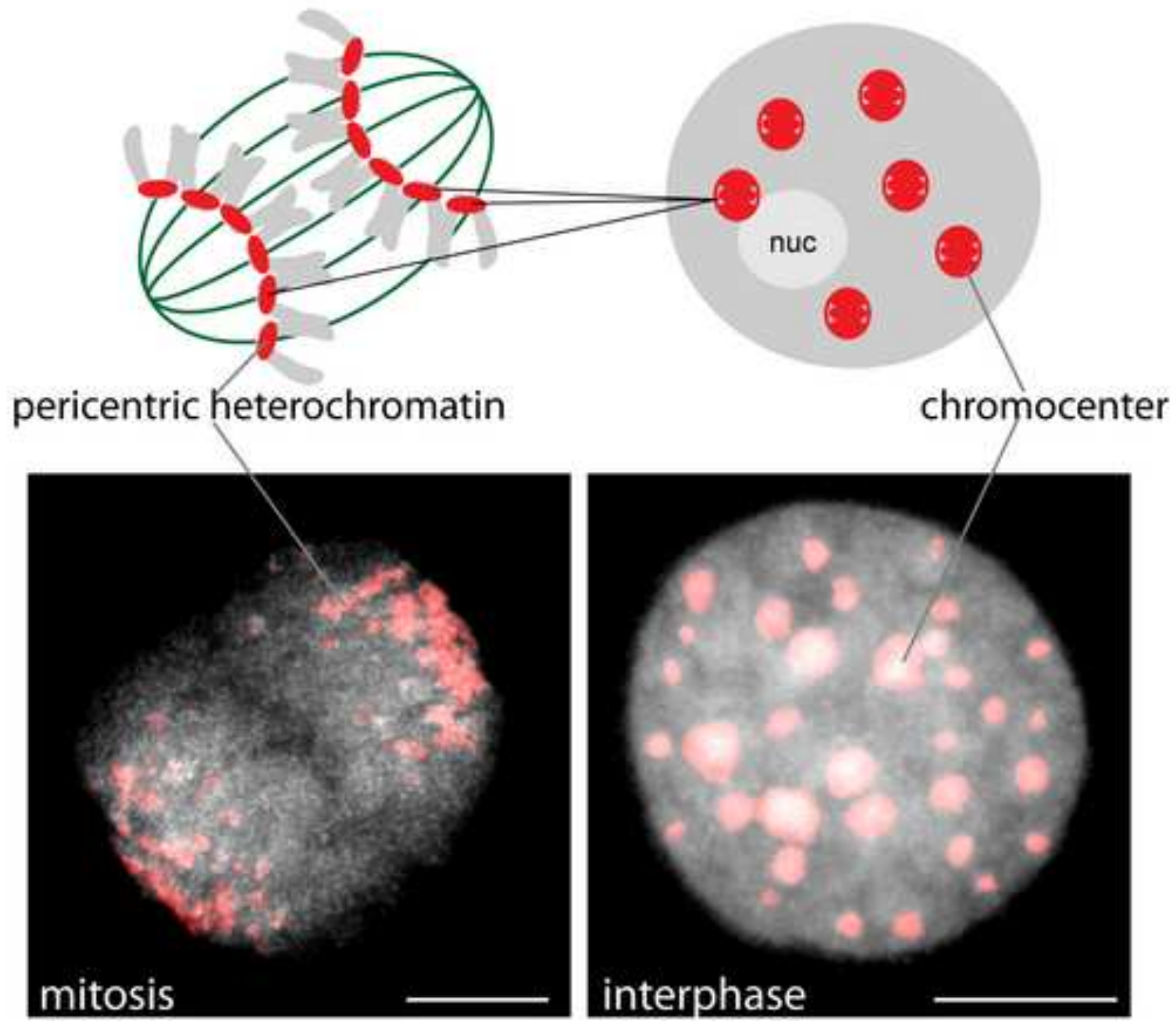




\section{(a) Somatic cells}

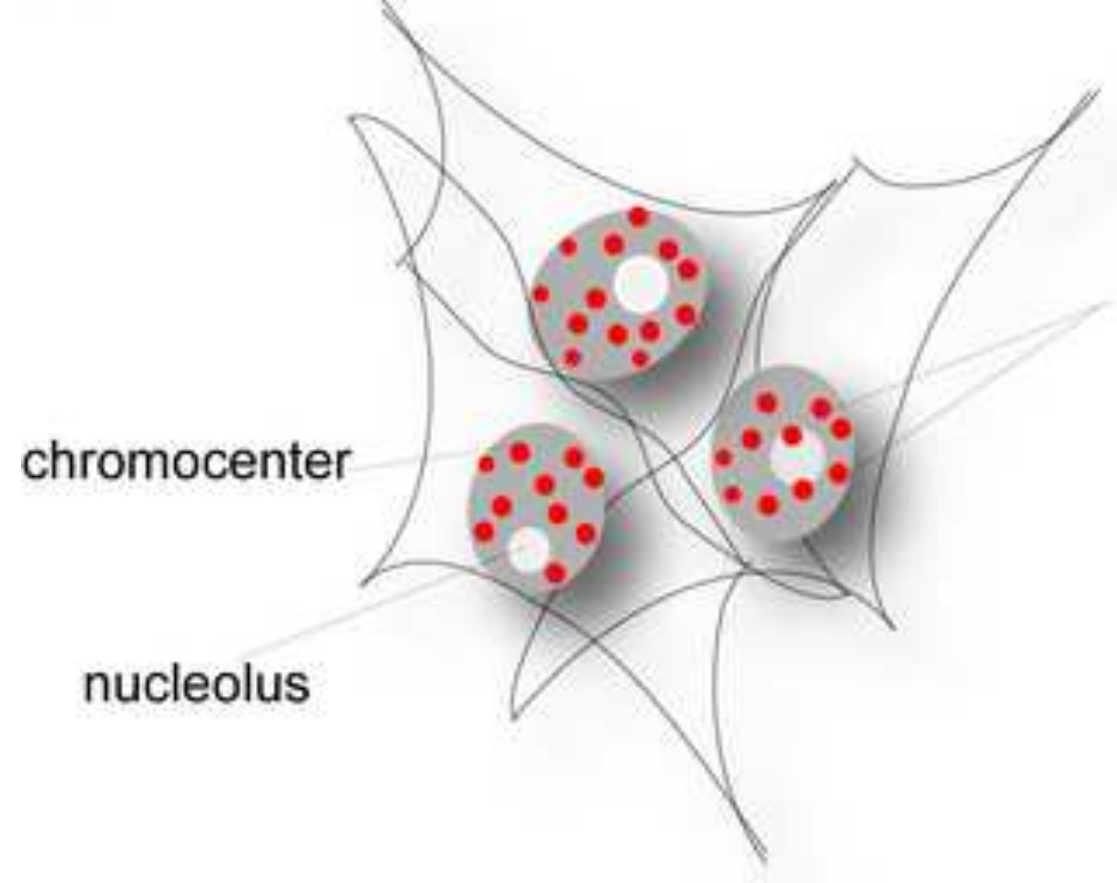

DNA methylation $\mathrm{H} 3 \mathrm{~K} 9 \mathrm{me} 2$ and $\mathrm{me} 3$

H3K27me1, H4K20me3

$\mathrm{H} 2 \mathrm{AZ}$

$\operatorname{HP} 1 \alpha, \beta$

structural RNA component

(b) Zygote

nucleolar-like body

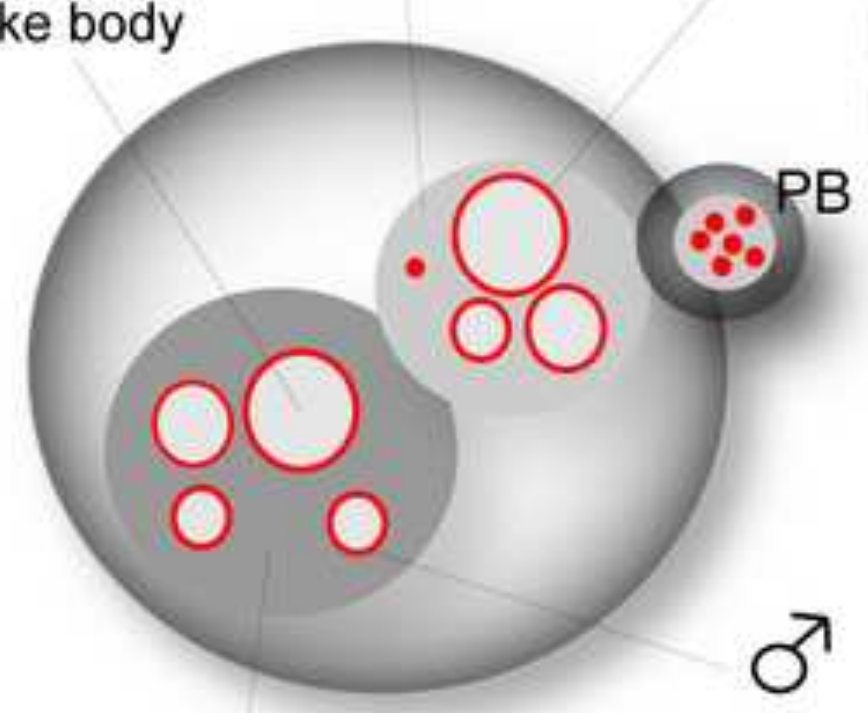

paternal pronucleus
DNA methylation $\mathrm{H} 3 \mathrm{~K} 9 \mathrm{me} 2$ and $\mathrm{me} 3$ H3K27me1, H4K20me3 H3K64me3

HP1 $\beta$

\section{DNA methylation}

万) $\mathrm{H} 3.3$

H3K9me1, H3K27me1 H3K27me3, PRC1 HP1ß 
Click here to download high resolution image

(a)

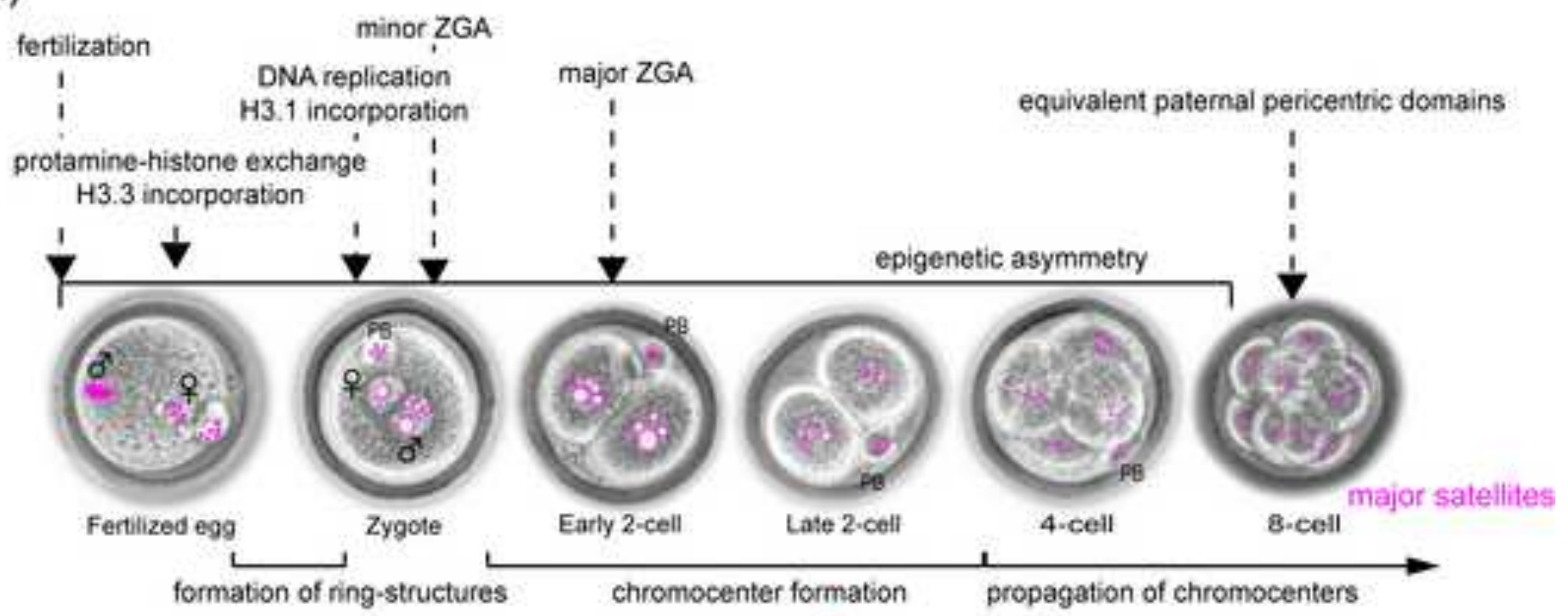

(b)
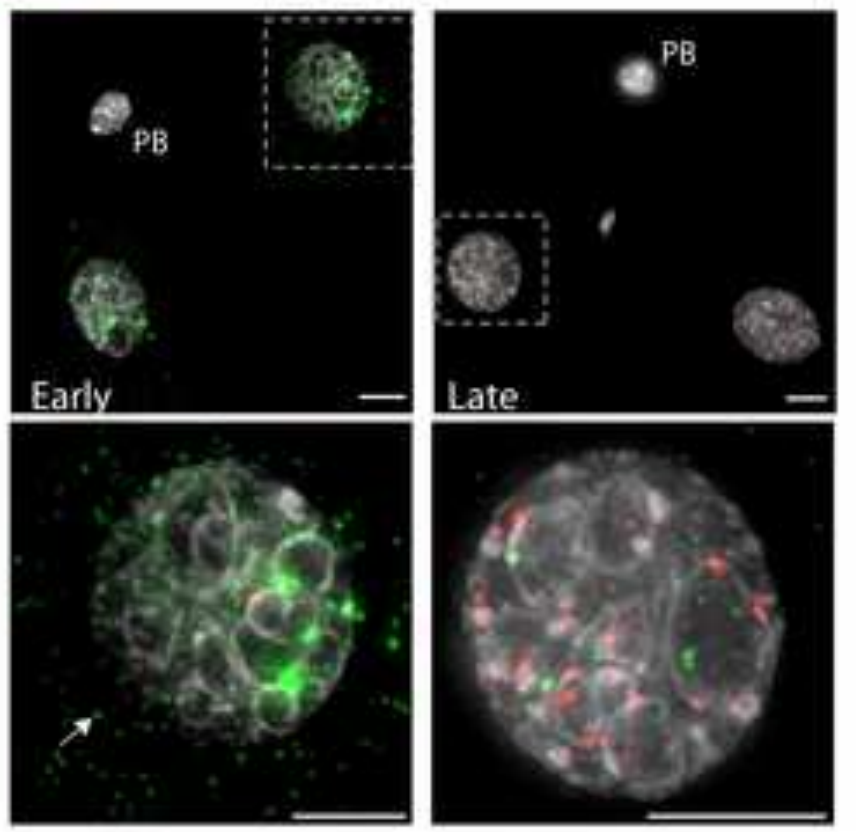

$$
10 \mu \mathrm{M}
$$

RNA FISH: Forward and Reverse major satellite transcripts 